\title{
Familial Aggregation of Migraine and Depression: Insights From a Large Australian Twin Sample
}

\author{
Yuanhao Yang, ${ }^{1}$ Huiying Zhao, ${ }^{1}$ Andrew C. Heath, ${ }^{2}$ Pamela A. F. Madden, ${ }^{2}$ Nicholas G. Martin, ${ }^{3}$ and \\ Dale R. Nyholt ${ }^{1}$ \\ ${ }^{1}$ Statistical and Genomic Epidemiology Laboratory, Institute of Health and Biomedical Innovation, Queensland University \\ of Technology, Brisbane, Queensland, Australia \\ ${ }^{2}$ Department of Psychiatry, Washington University School of Medicine, St. Louis, MO, USA \\ ${ }^{3}$ Genetic Epidemiology Laboratory, QIMR Berghofer Medical Research Institute, Brisbane, Queensland, Australia
}

Objectives: This research examined the familial aggregation of migraine, depression, and their cooccurrence.

Methods: Diagnoses of migraine and depression were determined in a sample of 5,319 Australian twins. Migraine was diagnosed by either self-report, the ID migraine ${ }^{T M}$ Screener, or International Headache Society (IHS) criteria. Depression was defined by fulfilling either major depressive disorder (MDD) or minor depressive disorder (MiDD) based on the Diagnostic and Statistical Manual of Mental Disorders (DSM) criteria. The relative risks (RR) for migraine and depression were estimated in co-twins of twin probands reporting migraine or depression to evaluate their familial aggregation and co-occurrence.

Results: An increased RR of both migraine and depression in co-twins of probands with the same trait was observed, with significantly higher estimates within monozygotic (MZ) twin pairs compared to dizygotic (DZ) twin pairs. For cross-trait analysis, the RR for migraine in co-twins of probands reporting depression was 1.36 (95\% Cl: 1.24-1.48) in MZ pairs and 1.04 (95\% Cl: 0.95-1.14) in DZ pairs; and the RR for depression in co-twins of probands reporting migraine was 1.26 (95\% Cl: $1.14-1.38)$ in $\mathrm{MZ}$ pairs and $1.02(95 \% \mathrm{Cl}$ : 0.94-1.11) in DZ pairs. The RR for strict IHS migraine in co-twins of probands reporting MDD was 2.23 (95\% Cl: 1.81-2.75) in MZ pairs and 1.55 (95\% Cl: 1.34-1.79) in DZ pairs; and the RR for MDD in co-twins of probands reporting IHS migraine was 1.35 (95\% Cl: 1.13-1.62) in $\mathrm{MZ}$ pairs and 1.06 (95\% Cl: 0.93-1.22) in DZ pairs.

Conclusions: We observed significant evidence for a genetic contribution to familial aggregation of migraine and depression. Our findings suggest a bi-directional association between migraine and depression, with an increased risk for depression in relatives of probands reporting migraine, and vice versa. However, the observed risk for migraine in relatives of probands reporting depression was considerably higher than the reverse. These results add further support to previous studies suggesting that patients with comorbid migraine and depression are genetically more similar to patients with only depression than patients with only migraine.

Keywords: migraine, depression, relative risk, familial aggregation, bi-directional association

Migraine, a recurrent disabling headache accompanied by chronic and episodic manifestations, is one of the most complex neurological disorders (Goadsby et al., 2002). Multiple cross-sectional studies (Ashina et al., 2012; Buse et al., 2010; Camarda et al., 2008; Fuller-Thomson et al., 2013; Mercante et al., 2005; Molgat \& Patten, 2005; Rist et al., 2013; Zwart et al., 2003) have consistently observed that migraine often co-occurs with depression, a psychological disorder characterized by dramatic decline in both mental and physical conditions (Remick, 2002).

\begin{abstract}
RECEIVED 31 March 2016; ACCEPTED 3 April 2016. First published online 6 June 2016.

ADDRESS FOR CORRESPONDENCE: Yuanhao Yang, Statistical and Genomic Epidemiology Laboratory, Institute of Health and Biomedical Innovation, Queensland University of Technology, GPO Box 2434, Brisbane QLD 4001, Australia. E-mail: yuanhao. yang@hdr.qut.edu.au
\end{abstract}


Also supported by some Caucasian population-based longitudinal studies (Breslau et al., 1994; Breslau et al., 2000; Breslau et al., 2003; Modgill et al., 2012), the association between migraine and depression is considered to be bi-directional, with migraine or severe headache increasing up to a 3-fold higher population relative risk (RR) of suffering depression, and vice versa. Another longitudinal study (Mongini et al., 2003) also revealed a potential bidirectional association between depression and migraine frequency and severity, while one U.S. study (Swartz et al., 2000) failed to repeat these results, possibly due to the influence of other comorbid psychological disorders, such as panic disorder, and social phobia, which were jointly analyzed in the study.

Although repeatedly observed, little is known about the mechanisms underlying the association between migraine and depression. An increased RR (i.e., RR > 1) of one trait, evaluated from twin and family samples, has been identified in relatives of probands reporting the same trait, for both migraine (Cologno et al., 2003; Kalfakis et al., 1996; Lemos et al., 2009; Russell \& Olesen, 1995; Stewart et al., 1997; Stewart et al., 2006; Thomsen et al., 2003) and depression (Barker et al., 2012; Merikangas et al., 2014; Schreier et al., 2006; Sullivan et al., 2000; Vandeleur et al., 2014). These results provide strong evidence on familial aggregation in migraineurs and depressive patients. The variation in estimated RRs among these studies is likely due to the different diagnostic approaches and study populations. For instance, the lifetime prevalence of migraine was calculated as 2028\% (Al-Hashel et al., 2014; Bicakci et al., 2008; Moens et al., 2007) based on the ID migraine ${ }^{\mathrm{TM}}$ Screener criteria (Lipton et al., 2003) but reduced to approximately $12 \%$ (Buse et al., 2012; Lipton et al., 2001) when based on the International Headache Society (IHS) criteria (Headache Classification Committee of the International Headache, 2013); and Caucasian populations had significant higher migraine and depression morbidity than African and Asian populations (Riolo et al., 2005; Stewart et al., 1996).

Significant familial aggregation of one trait indicates the presence of shared genetic and/or environmental factors in susceptibility of the trait. A considerable genetic contribution can be further determined after excluding environmental factors. Because both monozygotic (MZ) and dizygotic (DZ) twin pairs share similar living resources and environment, they are considered to experience the same environmental factors. Therefore, the observation of higher RRs within $\mathrm{MZ}$ twin pairs compared to $\mathrm{DZ}$ twin pairs provides evidence for a genetic contribution in susceptibility of the trait. For example, a significant higher RR for prostate cancer within male $\mathrm{MZ}$ pairs $(\mathrm{RR}=12.3,95 \% \mathrm{CI}=8.4-18.1)$ compared to male DZ pairs $(\mathrm{RR}=3.1,95 \% \mathrm{CI}=1.9-4.9$; Lichtenstein et al., 2000), indicates a significant contribution of genetic factors for the risk of prostate cancer.

The heritability of migraine and depression are both estimated at approximately 50\% (Levinson, 2006; Mulder et al., 2003), indicating both genetic and environmental factors play an important role in their development. Therefore, extending RR analyses in MZ and DZ twin pairs across both migraine and depression provides a natural experiment to determine the existence of shared genetic components between the two traits. Ours is the first such study to use a large population-based Australian twin sample to examine the association between migraine and depression.

In this article, we first examine the familial aggregation of migraine, depression, and their co-occurrence. We next compare relative risks estimated in $\mathrm{MZ}$ twin pairs to those estimated in DZ twin pairs to provide evidence for the contribution of genetic factors towards their risk.

\section{Materials and Methods}

\section{Samples}

As shown in Figure 1, participants were drawn from three Australian twin cohorts based at QIMR Berghofer Medical Research Institute (Heath et al., 2001; Wright \& Martin, 2004). Subjects with migraine and depression status were selected and constituted the 'merged migraine sample' $(N$ $=38,279)$ and 'merged depression sample' $(N=60,170)$ respectively. Definitions of migraine and depression were homogenized across the cohorts. Subjects also answered questions regarding demographic characteristics (e.g., sex, date of birth, zygosity) via semi-structured telephone interview and/or questionnaire. After combining the two merged samples and removal of non-twins and twins with missing status of either migraine or depression, a total of 5,319 twin pairs (2,456 MZ and 2,863 DZ pairs) remained for analysis.

\section{Assessment of Migraine}

Migraine symptom information ranged from single-answer self-report (yes or no) of migraine, using the ID Migraine ${ }^{\mathrm{TM}}$ Screener (Lipton et al., 2003) - three questions shown to accurately identify $93 \%$ of people with migraines - to detailed IHS diagnostic criteria (International Classification of Headache Disorders, ICHD-3; Headache Classification Committee of the International Headache, 2013). For the collection of detailed ICHD-3 diagnostic criteria (see Table 1), participants answering 'yes' to ever having 'migraine or recurrent attacks of headache' (screening positive), then answered a number of questions relating to their symptoms. Diagnoses were determined for the two major varieties of migraine: 1.1 migraine without aura (MO) and 1.2 migraine with aura (MA, primarily comprising 1.2.1 typical aura with migraine headache), which account for 90-95\% of all IHS migraines (Launer et al., 1999).

After careful merging of all available migraine information, lifetime diagnoses for migraine were made subject to data availability, according to: (1) IHS ICHD-3 MO/MA diagnostic criteria, (2) the ID Migraine ${ }^{\mathrm{TM}}$ Screener, or (3) self-reported migraine. Hence, migraine status was measured in four categories according to these three criteria: 


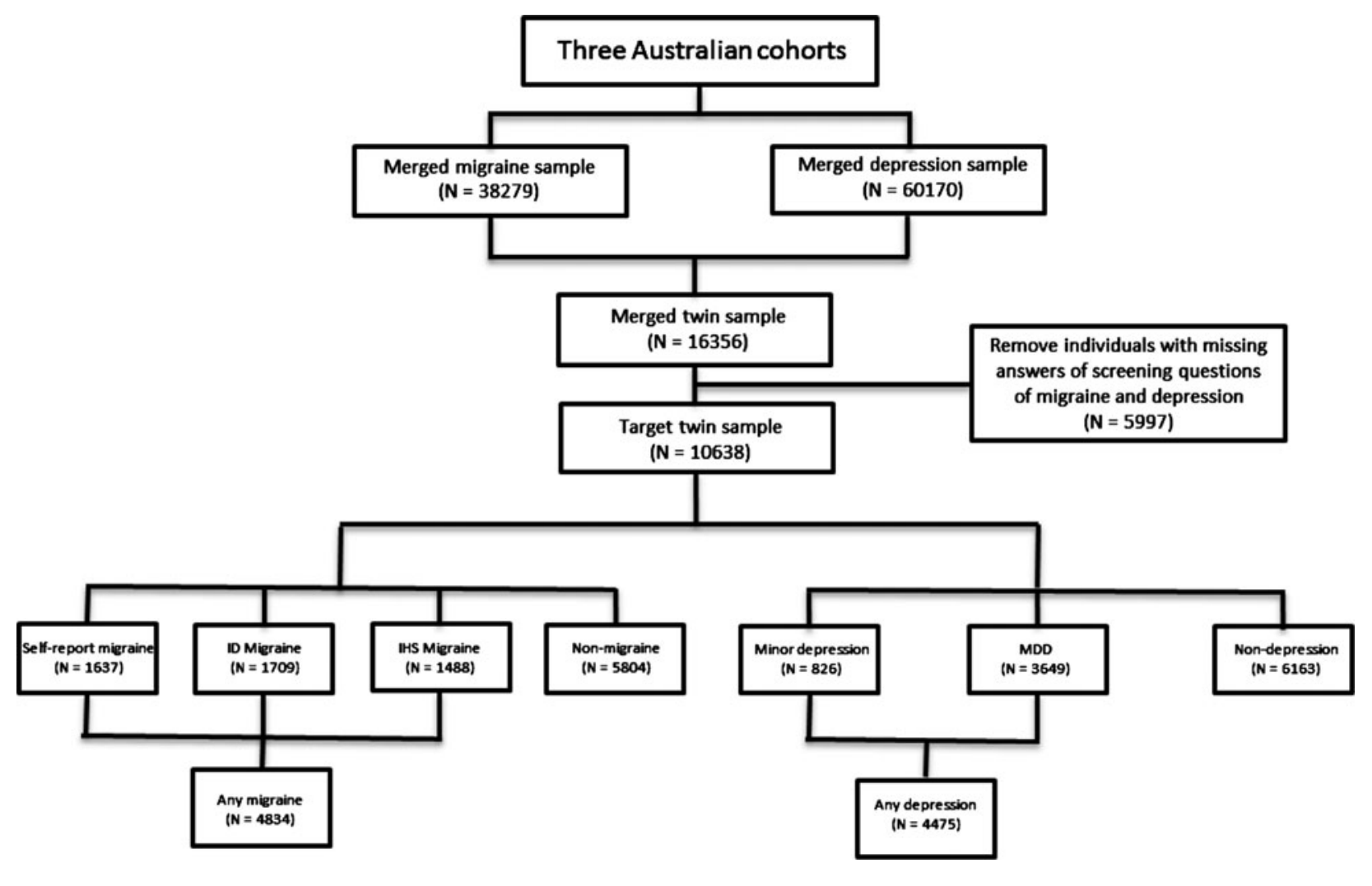

FIGURE 1

The flow chart of selected twin sample.

non-migraine, self-report migraine (i.e., participants with positive status of self-reported measurement but negative or unknown status of the other two criteria), ID migraine (i.e., participants with positive status ID migraine ${ }^{\mathrm{TM}}$ Screener criteria but negative or unknown IHS-based migraine status) and IHS migraine (i.e., participants with positive status of IHS-based migraine). The 'broad' migraine status (i.e., any migraine) was defined when participants had reported at least one positive migraine status; and the 'narrow' migraine status was strict to the IHS migraine status.

\section{Assessment of Depression}

Participants were first asked two screening questions: 'Has there ever been two weeks or more when you were depressed or down most of the day, nearly every day?' and 'Has there ever been two weeks or more when you were a lot less interested in most things or unable to enjoy the things you used to enjoy, most of the day, nearly every day?' With at least one positive response, participants then answered additional questions (see Table 1). Lifetime depression was diagnosed according to the third and revised edition of the Diagnostic and Statistical Manual of Mental Disorders (DSM-III-R; American Psychiatric Association, 1994) criteria: during a 2-week period, participants who had positive responses of more than five symptoms were diagnosed as suffering major depressive disorder (MDD) and participants who had 2-4 positive responses were diagnosed as suffering minor depressive disorder (MiDD). Therefore, in our study, depression status was measured in three categories: non-depression, MiDD, and MDD. Participants with either MiDD or MDD were defined to have the 'broad' depression status (i.e., any depression), and the MDD status was used as the 'narrow' depression status.

\section{Analyses}

Of the total 5,319 twin pairs included in the target sample, we stratified them into five subgroups according to sex and zygosity: female $M Z$ pairs, male $M Z$ pairs, female $D Z$ pairs, male DZ pairs, and opposite sex DZ pairs. RRs were calculated as the statistical ratio comparing the frequency of a target disorder occurring in co-twins of probands and that occurring in co-twins of health controls, which was calculated from the cross-tabulations of proband-co-twin pairs (see supplementary Tables S1-S6). RR for migraine and depression was estimated in co-twins of twin probands reporting migraine or depression to evaluate their familial aggregation and co-occurrence.

Age at onset for migraine and depression was not available, therefore the 'survey age', representing the age when subjects participated in the survey, was used to adjust for potential age effects. To investigate whether age would 
TABLE 1

The Survey Questions of IHS-Based Migraine and DSM-Based Depression

\begin{tabular}{|c|c|c|}
\hline Code & Abbreviation & Question \\
\hline \multicolumn{3}{|c|}{ IHS-based migraine } \\
\hline A & $\geq 5$ attacks & How many (migraine/episodes of headache) have you had during your lifetime? \\
\hline $\mathrm{B}$ & $\overline{4}-72$ hours & On average, how long (does/did) a typical (migraine/headache) episode? \\
\hline $\mathrm{C} 1$ & Unilateral location & Do the headaches usually occur on one side of the head? \\
\hline $\mathrm{C} 2$ & Pulsating quality & $\begin{array}{l}\text { Would you describe the headache pain you usually experience as: throbbing, } \\
\text { pulsating or pounding? }\end{array}$ \\
\hline C3 & Moderate/severe pain intensity & $\begin{array}{l}\text { Would you describe the pain associated with your headaches as: mild, } \\
\text { moderate, severe or unbearable? }\end{array}$ \\
\hline $\mathrm{C} 4$ & Aggravation by physical activity & $\begin{array}{l}\text { Are your headaches aggravated by walking up or down stairs or similar routine } \\
\text { physical activity? }\end{array}$ \\
\hline D1 & Nausea and/or vomiting & $\begin{array}{l}\text { Have you ever had recurrent attacks of any of the following: nausea, vomiting } \\
\text { or diarrhea? }\end{array}$ \\
\hline D2 & Photophobia and phonophobia & Do you experience enhanced sensitivity to light/smell? \\
\hline Aura_A & $\geq 2$ attacks with aura & $\begin{array}{l}\text { How many headaches with these kinds of aura symptoms have you had during } \\
\text { your lifetime? }\end{array}$ \\
\hline Aura_B & Visual/speech/sensory aura & $\begin{array}{l}\text { Have you ever had: visual disturbances lasting several minutes? Difficulties to } \\
\text { speak? One sided numbness or weakness? }\end{array}$ \\
\hline Aura_C1 & Spreads $\geq 5$ minutes & $\begin{array}{l}\text { How fast does your visual disturbance develop: the symptom is at its } \\
\text { worst/strongest right away (less than 1-2 minutes); or it gets worse/expands } \\
\text { after more than } 4 \text { minutes? }\end{array}$ \\
\hline Aura_C2 & Last $5-60$ minutes & $\begin{array}{l}\text { How long does your visual disturbance last: the symptom lasts less } \\
\text { than } 1 \text { minute; it lasts more than } 1 \text { minute but less than } 1 \text { hour; it lasts more } \\
\text { than } 1 \text { hour? }\end{array}$ \\
\hline Aura_C3 & Duration within 60 minutes & $\begin{array}{l}\text { How visual disturbances and headache connected in time are: headaches follow } \\
\text { the symptom within one hour; headaches do not follow the symptom until } \\
\text { later; headaches and symptom occur simultaneously; or headaches come } \\
\text { before the symptom? }\end{array}$ \\
\hline \multicolumn{3}{|c|}{ DSM-based depression } \\
\hline A1 & Depressed mood & $\begin{array}{l}\text { Has there ever been } 2 \text { weeks or more when you were depressed or down most } \\
\text { of the day, nearly every day? }\end{array}$ \\
\hline A2 & Loss of interest & $\begin{array}{l}\text { Has there ever been } 2 \text { weeks or more when you were a lot less interested in } \\
\text { most things or unable to enjoy the things you used to enjoy most of the day, } \\
\text { nearly every day? }\end{array}$ \\
\hline B1 & Weight/appetite change & Did you have a change in weight/appetite? \\
\hline B2 & Sleep disturbance & Did you have more trouble sleeping than usual? \\
\hline B3 & $\begin{array}{l}\text { Psychomotor } \\
\text { agitation/retardation }\end{array}$ & $\begin{array}{l}\text { Were you so fidgety or restless that you were unable to sit still?/Were you } \\
\text { talking or moving much more slowly than is normal for you? }\end{array}$ \\
\hline B4 & Fatigue/loss of energy & Were you feeling a loss of energy or more tired than usual? \\
\hline B5 & Worthlessness/feel guilty & $\begin{array}{l}\text { Were you feeling excessively guilty or that you were a bad person?/Were you } \\
\text { feeling that you were a failure or worthless? }\end{array}$ \\
\hline B6 & $\begin{array}{l}\text { Indecisiveness/diminish ability } \\
\text { to concentrate }\end{array}$ & $\begin{array}{l}\text { Were you having trouble thinking or concentrating?/Was it hard to make } \\
\text { decisions about everyday things? }\end{array}$ \\
\hline B7 & Suicide attempt & Were frequently thinking about death? \\
\hline
\end{tabular}

influence RR estimation, logistic regression analyses were performed to calculate the effect of age in four analyses (i.e., migraine in co-twins of probands reporting migraine, depression in co-twins of probands reporting depression, migraine in co-twins of probands reporting depression, and depression in co-twins of probands reporting migraine). Probands were randomly selected as the first interviewed twin for the same-sex MZ/DZ pairs; while for the opposite sex DZ pairs, RR estimates were obtained for selecting the female twin as well as the male twin as proband, to estimate cross-sex RRs.

We estimated RRs and their 95\% confidence intervals (CIs) for migraine (i.e., self-report migraine, ID migraine, IHS migraine, and any migraine) and depression (i.e., MDD and any depression) to assess their familial aggregation. We then separately estimated the RRs of migraine in co-twins of probands with depression and the RRs of depression in co-twins of probands with migraine, for broad diagnosis (i.e., any migraine and any depression) and narrow diag- nosis (i.e., IHS migraine and MDD) respectively. All the analyses were performed using either SPSS (v22) or Rstudio (RStudio Team, 2014).

\section{Results}

\section{Demographics}

As shown in Table 2, based on 10,638 individuals $(6,584$ females and 4,054 males) from the twin sample, the lifetime prevalence was estimated at $45 \%$ for any migraine and $42 \%$ for any depression. Consistent with the previous findings (Arroyo-Quiroz et al., 2014; Bierut et al., 1999; Buse et al., 2012; Kendler et al., 1992), the lifetime prevalence of IHS MO/MA migraine and DSM-III-R-based MDD was estimated at $14 \%$ and $34 \%$ respectively. Generally, females showed higher lifetime prevalence than males; the differences were small for selfreport migraine but increased for ID migraine and especially IHS migraine. Both any depression and MDD also 
TABLE 2

Lifetime Prevalence of Migraine and Depression Based on Australian Twin Sample

\begin{tabular}{|c|c|c|c|c|c|c|c|}
\hline \multirow[b]{2}{*}{ Lifetime prevalence } & \multicolumn{4}{|c|}{ Migraine } & \multicolumn{3}{|c|}{ Depression } \\
\hline & Self-report migraine & ID migraine & IHS migraine & Any-mig & MiDD & MDD & Any-dep \\
\hline Total sample & $15.39 \%$ & $16.07 \%$ & $13.99 \%$ & $45.44 \%$ & $7.76 \%$ & $34.30 \%$ & $42.07 \%$ \\
\hline Female & $15.98 \%$ & $19.58 \%$ & $17.68 \%$ & $53.24 \%$ & $7.50 \%$ & $37.68 \%$ & $45.19 \%$ \\
\hline Male & $14.43 \%$ & $10.36 \%$ & $7.99 \%$ & $32.78 \%$ & $8.19 \%$ & $28.81 \%$ & $37.00 \%$ \\
\hline
\end{tabular}

Note: Any-mig = Any migraine; Any-dep = Any depression.

demonstrated higher lifetime prevalence in females than in males, whereas the lifetime prevalence of MiDD was higher in males compared to females.

The mean 'survey age' was estimated at $36 \pm 11$ years, ranging from 18 to 89 years. The logistic regressions under all four analyses indicated that age does not significantly influence risk for migraine and depression in our sample $(p$ value $>.05$ ). Therefore, we present RRs without age adjustment.

\section{Familial Aggregation}

As shown in Table 3, we observed a significantly increased $\mathrm{RR}(\mathrm{RR}=1.73,95 \% \mathrm{CI}: 1.62-1.85)$ for any migraine in cotwins of probands reporting any migraine, compared with co-twins of non-migraine controls. The RR for any migraine was significantly higher within $\mathrm{MZ}$ pairs $(\mathrm{RR}=2.07,95 \%$ CI: $1.88-2.27$ ) compared to DZ pairs ( $\mathrm{RR}=1.47,95 \% \mathrm{CI}$ : 1.34-1.62). Similarly, regardless of migraine diagnosis (i.e., self-report migraine, ID migraine, IHS migraine, or any migraine), an increased RR was consistently found in the total sample, and a significant higher RR was calculated within MZ pairs compared to DZ pairs, although RRs calculated in $\mathrm{DZ}$ pairs for self-report migraine and ID migraine were not statistically significant, most likely due to their reduced diagnostic reliability and smaller sample size. In addition, most RRs calculated within same-sex DZ pairs were slightly higher than those calculated within DZ opposite-sex pairs, suggesting the presence of sex-specific effects in familial aggregation. Furthermore, RR for migraine increased in line with the reliability of migraine diagnosis, with no significant difference in RR between self-report migraine and ID migraine, but a significantly higher RR for IHS migraine. This pattern of results was observed in all zygosity subgroups.

Regarding depression, although any depression comprised two types of depression, MDD and MiDD, because of the relatively small number of MiDD patients $(N=826$ participants), we focused on results for MDD and any depression. Similar to migraine, an increased RR for depression in co-twins of probands reporting depression was observed for both MDD (RR $=1.75,95 \%$ CI: $1.63-1.89)$ and any depression ( $\mathrm{RR}=1.53,95 \% \mathrm{CI}: 1.43-1.63)$ in the total sample. These RRs significantly increased to 2.35 (95\% CI: 2.10-2.62) for MDD and 1.89 (95\% CI: 1.72-2.08) for any depression in MZ pairs, and significantly decreased to 1.36 (95\% CI: $1.23-1.51$ ) for MDD and 1.27 (95\% CI: 1.17-1.39) for any depression in DZ pairs, respectively. RRs calculated in all zygosity subgroups also remained significant, and a slightly higher RR was observed within same-sex DZ pairs compared to DZ pairs. Also similar to migraine, the RRs in DZ pairs for the narrow diagnosis (MDD) were higher although not statistically significant, compared to RRs for the broader any depression.

\section{Association Between Migraine and Depression}

For the broad diagnoses (Table 4) in the total sample, cotwins of probands reporting any depression had a significantly increased $\mathrm{RR}(\mathrm{RR}=1.18,95 \% \mathrm{CI}$ : $1.11-1.26$ ) for any migraine compared to co-twins of controls. The reverse was also true, with the RR for any depression significantly increased ( $\mathrm{RR}=1.12,95 \% \mathrm{CI}: 1.05-1.20)$ in co-twins of probands reporting any migraine compared to co-twins of controls, thus indicating a bi-directional association between the two disorders. The RR for any migraine in cotwins of probands reporting any depression was higher than the reverse. The bi-directional association became more significant within $M Z$ pairs, with an RR for any migraine in co-twins of probands reporting any depression of $1.36(95 \%$ CI: 1.24-1.48) and RR for any depression in co-twins of probands reporting any migraine was 1.26 (95\% CI: 1.141.38). Similar to some of the single-trait RR estimates, the cross-trait RR estimates decreased in DZ twin pairs and became non-significant, and RRs in same-sex DZ pairs were increased compared to opposite-sex DZ pairs. Also, significantly higher cross-trait RRs were observed within MZ pairs compared to DZ pairs for any migraine in co-twins of probands reporting any depression and vice versa, suggesting the presence of shared genetic components between any migraine and any depression.

For the narrow diagnoses (Table 4), a bi-directional association between IHS migraine and MDD was also found and was more significant compared to the broad diagnosis in the total sample and MZ pairs. The RRs for IHS migraine in co-twins of probands reporting MDD was 1.67 (95\% CI: 1.46-1.92) in the total sample and 2.23 (95\% CI: 1.81-2.75) in MZ pairs; while the RRs for MDD in cotwins of probands reporting IHS migraine was 1.25 (95\% CI: 1.13-1.37) in the total sample and 1.55 (95\% CI: 1.341.79) in MZ pairs. In contrast, results in DZ pairs suggest a unidirectional association, with a significant RR for IHS migraine in co-twins of probands reporting MDD at 1.35 


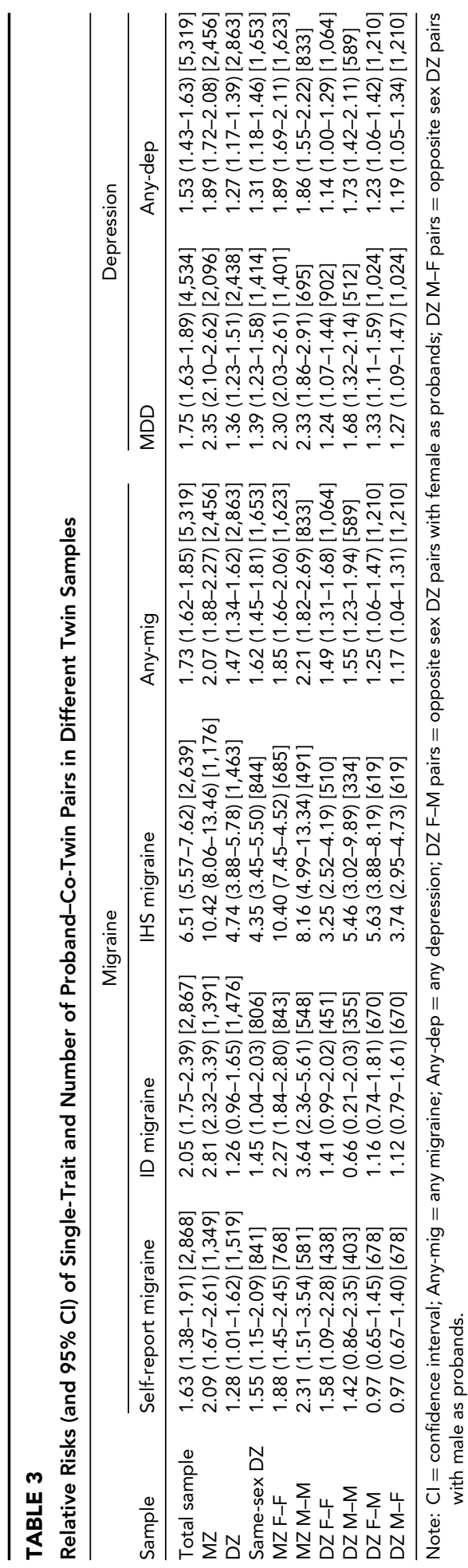

(95\% CI: 1.13-1.62) and a non-significant RR for MDD in co-twins of probands reporting IHS migraine at 1.06 (95\% CI: 0.93-1.22), respectively. Compared to DZ pairs, $\mathrm{MZ}$ pairs always provided significantly higher RRs for IHS migraine in co-twins of probands reporting MDD and the reverse. The difference in RRs calculated in $\mathrm{MZ}$ and DZ pairs was larger for the narrow diagnoses compared to the broad diagnoses. Notably, the observed risk for IHS migraine in co-twins of probands reporting MDD was considerably higher than the reverse, for total sample, MZ pairs and DZ pairs.

\section{Discussion}

\section{Familial Aggregation of Migraine and Depression?}

Previous Caucasian population-based family studies (Cologno et al., 2003; Kalfakis et al., 1996; Lemos et al., 2009; Russell \& Olesen, 1995; Stewart et al., 1997; Stewart et al., 2006; Thomsen et al., 2003) consistently reported familial aggregation of migraine in first-degree relatives of probands, with highly variable RRs ranging from 1.40 to 13.82, largely due to differences in migraine types (e.g., MA, familial hemiplegic migraine), diagnostic approaches (e.g., IHS-based self-report questionnaire or interview) and sample selection. Our results from DZ pairs are equivalent to proband-first-degree RR estimates from the general population, and are in line with previous RR estimates based on IHS migraine and any migraine status. By comparing our results for strict IHS migraine to results for the broader definitions of migraine, self-report migraine, and ID migraine status clearly demonstrates such expected sensitivity of RR and familial aggregation analysis of migraine to the diagnostic approach. Our finding of significantly higher RR for migraine within MZ pairs than DZ pairs provides strong evidence for a genetic contribution to migraine susceptibility.

Previous studies on measuring clustering of depression often utilized odds ratios (ORs) rather than RRs. The OR for depression in first-degree relatives of depressive probands have been calculated at approximately 2-3 in Caucasian population-based family samples (Barker et al., 2012; Merikangas et al., 2014; Schreier et al., 2006; Sullivan et al., 2000; Vandeleur et al., 2014). Assuming a depression prevalence of between $20 \%$ and $50 \%$, ORs ranging from 2 to 3 are estimated to be equivalent to RRs of approximately 1.11-1.50 (using OR to Risk Ratio Conversion; http://clincalc.com/Stats/ConvertOR.aspx). Thus, our RR estimates for depression from DZ pairs are in agreement with published findings, and support the familial aggregation of depression. Our finding of significantly higher RR for depression within $\mathrm{MZ}$ pairs compared to $\mathrm{DZ}$ pairs provides strong evidence for a genetic contribution to depression.

Although the difference in RRs between DZ F-M pairs (opposite sex DZ pairs with female as proband) and DZ M-F pairs (opposite sex DZ pairs with male as proband) 
TABLE 4

Relative Risks (and 95\% Cl) of Cross-Trait and Number of Proband-Co-Twin Pairs in Different Twin Samples

\begin{tabular}{|c|c|c|c|c|}
\hline \multirow{3}{*}{ Sample } & \multicolumn{4}{|c|}{ Proband-co-twin } \\
\hline & \multicolumn{2}{|c|}{ Broad diagnosis } & \multicolumn{2}{|c|}{ Narrow diagnosis } \\
\hline & Any-dep-Any-mig & Any-mig-Any-dep & MDD-IHS migraine & IHS migraine-MDD \\
\hline Total sample & $1.18(1.11-1.26)[5,319]$ & $1.12(1.05-1.20)[5,319]$ & $1.67(1.46-1.92)[3,450]$ & $1.25(1.13-1.37)[3,287]$ \\
\hline $\mathrm{MZ}$ & $1.36(1.24-1.48)[2,456]$ & $1.26(1.14-1.38)[2,456]$ & $2.23(1.81-2.75)[1,506]$ & $1.55(1.34-1.79)[1,473]$ \\
\hline DZ & $1.04(0.95-1.14)[2,863]$ & $1.02(0.94-1.11)[2,863]$ & 1.35 (1.13-1.62) [1,944] & $1.06(0.93-1.22)[1,814]$ \\
\hline Same-sex DZ & $1.07(0.96-1.19)[1,653]$ & $1.06(0.95-1.18)[1,653]$ & $1.39(1.12-1.71)[1,082]$ & $1.04(0.88-1.22)[1,068]$ \\
\hline MZ F-F & $1.28(1.16-1.40)[1,623]$ & $1.18(1.06-1.32)[1,623]$ & 2.19 (1.75-2.75) [934] & $1.42(1.21-1.66)$ [925] \\
\hline MZ M-M & $1.52(1.25-1.86)[833]$ & $1.30(1.08-1.56)[833]$ & $1.69(0.99-2.88)[572]$ & $1.46(0.99-2.17)[548]$ \\
\hline DZ F-F & $1.01(0.90-1.13)[1,064]$ & $1.03(0.90-1.16)[1,064]$ & $1.17(0.94-1.46)[668]$ & $0.94(0.78-1.13)[666]$ \\
\hline DZ M-M & $1.08(0.85-1.37)$ [589] & $0.99(0.80-1.23)[589]$ & $1.81(1.09-3.00)[414]$ & $1.17(0.78-1.75)[402]$ \\
\hline DZ F-M & $1.02(0.87-1.20)[1,210]$ & $0.96(0.83-1.12)[1,210]$ & $1.38(0.97-1.98)$ [862] & $1.10(0.88-1.38)[746]$ \\
\hline DZ M-F & $0.97(0.86-1.09)[1,210]$ & $1.02(0.89-1.16)[1,210]$ & $1.12(0.86-1.45)[746]$ & $1.22(0.99-1.50)[862]$ \\
\hline
\end{tabular}

$\mathrm{M}-\mathrm{F}$ pairs = opposite sex DZ pairs with male as probands.

for migraine and depression are not significant, the risks for migraine and depression are consistently larger in male co-twins of affected female probands compared to female co-twins of affected male probands. These results suggest the possible influence of sex-specific effects (i.e., effects expressed in one sex but not the other) that may include genetic $(G)$, environmental $(E)$, and/or interacting $(G \times E)$ effects.

\section{Bi-Direction Association Between Migraine and De- pression?}

In line with previous findings (Breslau et al., 1994; Breslau et al., 2000; Breslau et al., 2003; Modgill et al., 2012; Mongini et al., 2003), results from analysis of broad diagnoses in the total sample indicate a bi-directional association between migraine and depression, with an increased RR for any migraine in co-twins of probands reporting any depression, and vice versa. The association became stronger when analyzing the more narrow diagnoses of IHS migraine and MDD.

The observed risk for migraine in relatives of probands reporting depression was considerably higher than the risk for depression in relatives of probands reporting migraine. These results were observed for both broad and narrow diagnoses, and remained when calculating RRs by averaging over selecting either twin 1 or twin 2 as proband, thus ensuring our results are robust to proband selection within twin pairs. These findings provide further support to findings from a recent analysis of single nucleotide polymorphism (SNP) genotype data indicating that patients with comorbid migraine and depression are genetically more similar to patients with only depression than patients with only migraine (Ligthart et al., 2014).

Regardless of the diagnostic approach, the association between migraine and depression was stronger within MZ twin pairs compared to DZ pairs, thus providing strong evidence for a genetic contribution to familial aggregation of migraine and depression.

\section{Strengths and Weaknesses}

Ours is the only study to use a large population-based twin design to provide strong evidence for a genetic contribution to the familial aggregation and co-occurrence of migraine and depression. Another advantage of this study concerned the utilization of multiple approaches to diagnose migraine and depression. Comparing results from different diagnostic definitions allowed us to demonstrate both the sensitivity and validity of RR and familial aggregation analysis of migraine and depression across different diagnostic criteria.

However, there are some limitations to note. First and foremost, both migraine and depression status were diagnosed using self-reported questionnaire data, as opposed to the gold-standard of clinical-based interviews by neurologists or psychologists. Although our approach may result in some misclassification of migraine and depression status, it is not feasible to perform clinic-based interviews in samples larger enough to provide sufficient power for such familial aggregation studies. Moreover, our approach enabled narrow diagnoses of migraine and depression that satisfy clinically accepted criteria. Furthermore, our estimated lifetime prevalence of IHS migraine and DSM-IIIR-based MDD are in a good agreement with published estimates (Arroyo-Quiroz et al., 2014; Bierut et al., 1999; Buse et al., 2012; Kendler et al., 1992). Second, we did not separate IHS migraine into MO and MA. However, given our previous findings indicating a strong familial aggregation and genetic overlap between MO and MA, we believe our approach provides the most powerful and sensible use of the data to examine the relationship between migraine and depression. Third, probands of twin pairs were selected randomly as the twin who first entered the survey rather than birth order. However, considering twins essentially share the same family environment and the fact we obtain identical RRs and conclusions when selecting the second (other) twin as the proband indicates our random selection approach to be valid. Another limitation relates to our use of 'survey age' rather than age of onset. However, given our 
samples were comprised of adults past the typical age of onset, and our logistic regression analyses found no association between survey age and migraine and/or depression, we do not believe this to be an important issue.

\section{Conclusions}

In summary, this study used a large Australian populationbased twin sample and found significant evidence for a genetic contribution to the familial aggregation of migraine and depression. Our findings also suggest a bi-directional association between migraine and depression, with an increased risk for depression in relatives of probands reporting migraine and vice versa. However, the observed risk for migraine in relatives of probands reporting depression was considerably higher than the reverse. These results add further support to previous studies suggesting that patients with comorbid migraine and depression are genetically more similar to patients with depression compared to patients with migraine.

A better understanding of the genetic architecture of comorbid migraine and depression has excellent potential to improve our understanding of the relationship between migraine and co-occurring depression and inform treatment strategies. For example, identifying patients most at risk of comorbid migraine and depression and chronic migraine (due to medication overuse), and identifying subgroups of patients for whom a particular treatment or treatment combination may be most effective (e.g., episodic vs. chronic migraine, depressed vs. non-depressed, those with high analgesic use). Hence, future studies should focus on characterizing and identifying the genetic and environmental factors contributing to co-occurring migraine and depression.

\section{Acknowledgments}

Huiying Zhao was supported by a National Health and Medical Research Council (NHMRC) Early Career Fellowship (APP1091816). Dale R. Nyholt was supported by an NHMRC Research Fellowship (APP0613674). This work was supported by an NHMRC project grant (APP1075175), the European Union's Seventh Framework program (20072013) under grant agreement no. 602633 (EUROHEADPAIN) and the US National Institutes of Health (AA07535, AA011998, AA017688, AA10249, AA13320, AA13321, AA13326, AA14041, MH66206, DA12854, DA019951).

\section{Supplementary Material}

To view supplementary material for this article, please visit http://dx.doi.org/10.1017/thg.2016.43.

\section{References}

Al-Hashel, J. Y., Ahmed, S. F., Alroughani, R., \& Goadsby, P. J. (2014). Migraine among medical students in Kuwait University. Journal of Headache and Pain, 15, 26.
American Psychiatric Association. (1994). Diagnostic and statistical manual of mental disorders (4th ed.). Washington, DC: Author.

Arroyo-Quiroz, C., Kurth, T., Cantu-Brito, C., Lopez-Ridaura, R., Romieu, I., \& Lajous, M. (2014). Lifetime prevalence and underdiagnosis of migraine in a population sample of Mexican women. Cephalalgia, 34, 1088-1092.

Ashina, S., Serrano, D., Lipton, R. B., Maizels, M., Manack, A. N., Turkel, C. C., ... Buse, D. C. (2012). Depression and risk of transformation of episodic to chronic migraine. Journal of Headache and Pain, 13, 615-624.

Barker, E. D., Copeland, W., Maughan, B., Jaffee, S. R., \& Uher, R. (2012). Relative impact of maternal depression and associated risk factors on offspring psychopathology. British Journal of Psychiatry, 200, 124-129.

Bicakci, S., Bozdemir, N., Over, F., Saatci, E., \& Sarica, Y. (2008). Prevalence of migraine diagnosis using ID Migraine among university students in southern Turkey. Journal of Headache and Pain, 9, 159-163.

Bierut, L. J., Heath, A. C., Bucholz, K. K., Dinwiddie, S. H., Madden, P. A., Statham, D. J., ... Martin, N. G. (1999). Major depressive disorder in a community-based twin sample: Are there different genetic and environmental contributions for men and women? Archives of General Psychiatry, 56, 557-563.

Breslau, N., Davis, G. C., Schultz, L. R., \& Peterson, E. L. (1994). Joint 1994 wolff award presentation. Migraine and major depression: A longitudinal study. Headache, 34, 387393.

Breslau, N., Lipton, R. B., Stewart, W. F., Schultz, L. R., \& Welch, K. M. (2003). Comorbidity of migraine and depression: Investigating potential etiology and prognosis. Neurology, 60, 1308-1312.

Breslau, N., Schultz, L. R., Stewart, W. F., Lipton, R. B., Lucia, V. C., \& Welch, K. M. (2000). Headache and major depression: Is the association specific to migraine? Neurology, 54, 308-313.

Buse, D. C., Manack, A., Serrano, D., Turkel, C., \& Lipton, R. B. (2010). Sociodemographic and comorbidity profiles of chronic migraine and episodic migraine sufferers. Journal of Neurology, Neurosurgery, \& Psychiatry, 81, 428-432.

Buse, D. C., Manack, A. N., Fanning, K. M., Serrano, D., Reed, M. L., Turkel, C. C., \& Lipton, R. B. (2012). Chronic migraine prevalence, disability, and sociodemographic factors: Results from the American Migraine prevalence and prevention study. Headache, 52, 1456-1470.

Camarda, C., Pipia, C., Taglialavori, A., Di Fiore, P., Camarda, R., \& Monastero, R. (2008). Comorbidity between depressive symptoms and migraine: Preliminary data from the Zabut Aging Project. Neurological Sciences, 29, S149-S151.

Cologno, D., De Pascale, A., \& Manzoni, G. C. (2003). Familial occurrence of migraine with aura in a population-based study. Headache, 43, 231-234.

Fuller-Thomson, E., Schrumm, M., \& Brennenstuhl, S. (2013). Migraine and despair: Factors associated with depression and suicidal ideation among Canadian migraineurs in a population-based study. Depression Research and Treatment, 2013, Article ID 401487. 
Goadsby, P. J., Lipton, R. B., \& Ferrari, M. D. (2002). Migraine current understanding and treatment. New England Journal of Medicine, 346, 257-270.

Headache Classification Committee of the International Headache Society. (2013). The international classification of headache disorders, 3rd edition (beta version). Cephalalgia, 33, 629-808.

Heath, A. C., Howells, W., Kirk, K. M., Madden, P. A., Bucholz, K. K., Nelson, E. C., ... Martin, N. G. (2001). Predictors of non-response to a questionnaire survey of a volunteer twin panel: Findings from the Australian 1989 twin cohort. Twin Research, 4, 73-80.

Kalfakis, N., Panas, M., Vassilopoulos, D., \& MalliaraLoulakaki, S. (1996). Migraine with aura: Segregation analysis and heritability estimation. Headache, 36, 320-322.

Kendler, K. S., Neale, M. C., Kessler, R. C., Heath, A. C., \& Eaves, L. J. (1992). A population-based twin study of major depression in women. The impact of varying definitions of illness. Archives of General Psychiatry, 49, 257-266.

Launer, L. J., Terwindt, G. M., \& Ferrari, M. D. (1999). The prevalence and characteristics of migraine in a populationbased cohort: The GEM study. Neurology, 53, 537-542.

Lemos, C., Castro, M. J., Barros, J., Sequeiros, J., PereiraMonteiro, J., Mendonca, D., \& Sousa, A. (2009). Familial clustering of migraine: Further evidence from a Portuguese study. Headache, 49, 404-411.

Levinson, D. F. (2006). The genetics of depression: A review. Biological Psychiatry, 60, 84-92.

Lichtenstein, P., Holm, N. V., Verkasalo, P. K., Iliadou, A., Kaprio, J., Koskenvuo, M., ... Hemminki, K. (2000). Environmental and heritable factors in the causation of cancer - Analyses of cohorts of twins from Sweden, Denmark, and Finland. New England Journal of Medicine, 343, 78-85.

Ligthart, L., Hottenga, J. J., Lewis, C. M., Farmer, A. E., Craig, I. W., Breen, G., .. . Nyholt, D. R. (2014). Genetic risk score analysis indicates migraine with and without comorbid depression are genetically different disorders. Human Genetics, 133, 173-186.

Lipton, R. B., Dodick, D., Sadovsky, R., Kolodner, K., Endicott, J., Hettiarachchi, J., Harrison, W., \& ID Migraine Validation Study. (2003). A self-administered screener for migraine in primary care: The ID Migraine Validation Study. Neurology, 61, 375-382.

Lipton, R. B., Stewart, W. F., Diamond, S., Diamond, M. L., \& Reed, M. (2001). Prevalence and burden of migraine in the United States: Data from the American Migraine Study II. Headache, 41, 646-657.

Mercante, J. P., Peres, M. F., Guendler, V., Zukerman, E., \& Bernik, M. A. (2005). Depression in chronic migraine: Severity and clinical features. Arquivos de Neuro-Psiquiatria, 63, 217-220.

Merikangas, K. R., Cui, L., Heaton, L., Nakamura, E., Roca, C., Ding, J., ... Angst, J. (2014). Independence of familial transmission of mania and depression: Results of the NIMH family study of affective spectrum disorders. Molecular Psychiatry, 19, 214-219.
Modgill, G., Jette, N., Wang, J. L., Becker, W. J., \& Patten, S. B. (2012). A population-based longitudinal community study of major depression and migraine. Headache, 52, 422-432.

Moens, G., Johannik, K., Verbeek, C., \& Bulterys, S. (2007). The prevalence and characteristics of migraine among the Belgian working population. Acta Neurologica Belgica, 107, 84-90.

Molgat, C. V., \& Patten, S. B. (2005). Comorbidity of major depression and migraine - A Canadian population-based study. Canadian Journal of Psychiatry, 50, 832-837.

Mongini, F., Keller, R., Deregibus, A., Raviola, F., Mongini, T., \& Sancarlo, M. (2003). Personality traits, depression and migraine in women: A longitudinal study. Cephalalgia, 23, 186-192.

Mulder, E. J., Van Baal, C., Gaist, D., Kallela, M., Kaprio, J., Svensson, D. A., ... Palotie, A. (2003). Genetic and environmental influences on migraine: A twin study across six countries. Twin Research, 6, 422-431.

Remick, R. A. (2002). Diagnosis and management of depression in primary care: A clinical update and review. CMAJ, 167, 1253-1260.

Riolo, S. A., Nguyen, T. A., Greden, J. F., \& King, C. A. (2005). Prevalence of depression by race/ethnicity: Findings from the national health and nutrition examination survey III. American Journal of Public Health, 95, 998-1000.

Rist, P. M., Schurks, M., Buring, J. E., \& Kurth, T. (2013). Migraine, headache, and the risk of depression: Prospective cohort study. Cephalalgia, 33, 1017-1025.

RStudio Team. (2014). RStudio: Integrated development for $R$. Boston, MA: Author. Retrieved from http://www. RStudio.com/ide.

Russell, M. B., \& Olesen, J. (1995). Increased familial risk and evidence of genetic factor in migraine. BMJ, 311, 541-544.

Schreier, A., Hofler, M., Wittchen, H. U., \& Lieb, R. (2006). Clinical characteristics of major depressive disorder run in families - A community study of 933 mothers and their children. Journal of Psychiatric Research, 40, 283-292.

Stewart, W. F., Bigal, M. E., Kolodner, K., Dowson, A., Liberman, J. N., \& Lipton, R. B. (2006). Familial risk of migraine: Variation by proband age at onset and headache severity. Neurology, 66, 344-348.

Stewart, W. F., Lipton, R. B., \& Liberman, J. (1996). Variation in migraine prevalence by race. Neurology, 47, 52-59.

Stewart, W. F., Staffa, J., Lipton, R. B., \& Ottman, R. (1997). Familial risk of migraine: A population-based study. Annals of Neurology, 41, 166-172.

Sullivan, P. F., Neale, M. C., \& Kendler, K. S. (2000). Genetic epidemiology of major depression: Review and meta-analysis. American Journal of Psychiatry, 157, 1552-1562.

Swartz, K. L., Pratt, L. A., Armenian, H. K., Lee, L. C., \& Eaton, W. W. (2000). Mental disorders and the incidence of migraine headaches in a community sample: Results from the Baltimore Epidemiologic Catchment area follow-up study. Archives of General Psychiatry, 57, 945-950. 
Thomsen, L. L., Olesen, J., \& Russell, M. B. (2003). Increased risk of migraine with typical aura in probands with familial hemiplegic migraine and their relatives. European Journal of Neurology, 10, 421-427.

Vandeleur, C. L., Merikangas, K. R., Strippoli, M. P., Castelao, E., \& Preisig, M. (2014). Specificity of psychosis, mania and major depression in a contemporary family study. Molecular Psychiatry, 19, 209-213.
Wright, M. J., \& Martin, N. G. (2004). Brisbane adolescent twin study: Outline of study methods and research projects. Australian Journal of Psychology, 56, 65-78.

Zwart, J. A., Dyb, G., Hagen, K., Odegard, K. J., Dahl, A. A., Bovim, G., \& Stovner, L. J. (2003). Depression and anxiety disorders associated with headache frequency. The NordTrondelag Health Study. European Journal of Neurology, 10, 147-152. 